In some of the cases the head is tilted owing to a further fissured fracture. In most cases it is advisable to limit the operation to removal of the loose fragment.

(3) In comminuted fractures the total circumference of the head is increased, by separation of the fragments. The operation of removal of the head and part of neck of the radius is necessary. My experience is that the best results are obtained by immobilization of the elbow for six weeks and then to operate.

If the arm is left still, ossification will not take place in the capsule during the waiting period, nor after the operation. As movement has not been allowed, there is no reason to suppose osteoarthritis will be more frequent in consequence of the delay, although it is probably inevitable in a few cases following any operation involving opening a joint. After this operation a full range of movement has been obtained, but usually extension is permanently limited by twenty degrees and other movements are full.

\section{Fractures of the Neck of the Radius}

These are usually transverse. A wedge-shaped piece of bone is often torn off the shaft from one side and is adherent to the head. Before the epiphysis has joined, the fracture is just distal to the cartilaginous plate, but the diaphyseal portion broken off is usually less than half the circumference of the neck.

(1) As a rule there is little displacement, or this may be a rotatory one. With little rotation or displacement, the treatment is similar to that of a fissured fracture of the head.

(2) In one-third of these fractures there was displacement, that is - a tilt to a considerable degree or displacement of the head right off the shaft, for it may be alongside the shaft, or be displaced to the inner side of the forearm. Operative treatment is indicated. Should the head be displaced as a whole, but also be broken into a number of fragments, it ought to be removed. In other cases reposition is possible and definitely indicated in patients under forty. In children it is very desirable, as removal of the head leads to cubitus valgus of extreme degree. This may be complicated by limited wrist-joint movement, radial deviation of the hand and prominence of the head of the ulna. The operation should be carried out within a week of the injury. If the head will stay in place after reposition, no fixation is necessary. If it will not remain in position it is often possible to fix it by a stitch of catgut through a tag of periosteum adherent to the neck or by passing a tiny needle through two drill holes distal to articular cartilage. In children the head may not stay in place without further fixation. I have reported a method of fixation by driving a bone peg through the articular surface of the head and up the shaft, so that at the end of the operation the peg was extra-articular and there was a minimum of damage to articular cartilage. Although the operation is difficult at times, I have continued to employ it with satisfactory results. I have seen on two occasions manipulative reposition of the head of the radius in children, when it had been displaced right off the shaft.

\section{Fractures on Outer Side of Lower End of Humerus}

In discussing fractures on the outer side of the lower end of the humerus, I am not including any which extend to the inner side, nor the comminuted fractures seen in adults associated with dislocation and fracture of the olecranon. Intra-articular fractures of the capitellum only appear to be very rare.

(1) In adults the line of fracture may be oblique from above the external epicondyle into the joint medial to the capitellum. More frequently the capitellum, part of the trochlea, and a piece of non-articular bone above and behind are fractured from the shaft. If there is no displacement, the elbow is put at rest at a right angle in a posterior plaster trough and $x$-rayed in the plaster to see that the position is still satisfactory. When there is displacement, open operation is indicated. Reposition of the fragments is not difficult. In most cases fixation is not required, but if the fragments will not remain in place, a stitch passed through a drill hole made in the diaphysis will often hold the fragments, or a small bone peg an eighth of an inch across may be driven transversely through the fragment to hold it to the trochlea.

(2) In children the fractured piece consists of the capitellar epiphysis (that is, the capitellum and half the trochlea) and a flake of the diaphysis, usually off the back of the lower end of the shaft. When there is no displacement the fracture is treated after the manner of a supracondylar fracture with no displacement. When there is displacement, an open reposition is employed or non-union with cubitus valgus will occur. Formerly one used some form of peg or nail fixation, but this is seldom necessary. A catgut stitch through the diaphyseal flake to periosteum or muscle on the shaft will hold it in place. Some fixation is necessary or else the fragment will displace laterally. Normal elbows are obtained after efficient reposition. The cases of fracture of this area in childhood and young adults which have been neglected not only lead to a weak elbow, but sometimes ulnar nerve neuritis or paralysis.

(3) Fractures of the external epicondyle are isolated and uncommon injuries. Relaxation of the muscles by flexing the elbow until union has occurred is sufficient treatment.

Of the last 100 consecutive cases of fracture on the outer side of the elbow-joint, open operations have been performed on :

Head of Radius (8 cases):

1. For single loose fragments, 3 cases (fragment removed).

2. For comminuted fracture, $\mathbf{5}$ cases (head removed in 3 cases, pieces in 2).

Neck of Radius (6 cases):

1. Removal of head, 1 case.

2. Reposition of head with fixation: (a) by catgut or none, 3 cases ; (b) by bone peg, 2 cases.

Humerus (5 cases)

1. Capitellum, 3 cases

2. External condyle in adult, 1 case.

3. One operation for ulnar nerve paralysis.

\section{FRACTURES OF THE INTERNAL EPICONDYLE OF THE HUMERUS*}

BY

S. L. HIGGS, M.B., B.Ch.Cantab., F.R.C.S.

SURGEON, ROYAL NATIONAL ORTHOPAEDIC HOSPITAL; ASSISTANT

SURGEON, ORTHOPAEDIC DEPARTMENT, ST. BARTHOLOMEW'S HOSPITAL

(With Special Plate)

In 100 consecutive cases of recent fractures of the elbowjoint treated at St. Bartholomew's Hospital sixteen involved the internal epicondyle of the humerus, and this represents about the average recorded in other statistics. The average age was 12 years. It is therefore not a rare injury. From the number of patients who seek advice on account of late complications it is clear that recognition and treatment of the original condition are often at fault.

\section{General Considerations}

The anatomical peculiarities of the internal epicondyle make it prone to injury in childhood. It has its own centre of ossification, which appears at the fifth year, and does not fuse until about the age of 16 . During this period separation of the epicondyle may occur through the relatively weak epiphyseal cartilage. To the epicondyle are attached the internal lateral ligament and the common origin of the flexor group of forearm muscles. Thus a

* Read in opening a discussion in the Section of Orthopaedics at the Annual Meeting of the British Medical Association, Oxford, 1936. 
forcible abduction of the joint acting through these structures may cause avulsion of the epicondyle with or without dislocation of the joint.

The chief importance of this fracture is on account of the close association of the epicondyle with the ulnar nerve, which is frequently involved either at the time or later. Apart from the serious complication of ulnar paralysis, inadequate treatment may lead to non-union of the epicondyle, some limitation of movement, and lateral instability of the joint.

Clinically it may not be easy to detect the nature of the injury. An $x$-ray examination is most necessary, but interpretation may be difficult unless the appearance of the ossific centres at various ages is familiar. Films taken of the other elbow for comparison will be of great value. Before the age of $\mathbf{5}$ the epicondyle may be completely avulsed without radiographic changes.

\section{Types and Treatment}

(a) A chip off the Internal Epicondyle or Fragmentation without Displacement.-Such cases, which are quite common, all do well with conservative treatment. A " collar and cuff " sling for two weeks and then gradual active movements will lead to complete recovery.

(b) Fractures of the Internal Epicondyle with Downward Displacement.-This group constitutes about half the cases. The epiphysis has been avulsed and the strong pull of the flexor muscles maintains the displacement. If the swelling it not too great the epicondyle can be felt as a movable mass much larger than the $x$-ray appearance would suggest, lying below and in front of its normal situation. There is undue lateral mobility owing to the internal lateral ligament becoming detached with the epicondyle. The routine treatment is to keep the elbow fixed in flexion, but this does not achieve reduction of the fracture or subsequent union. The immediate results appear satisfactory, but these cases may be seen again in later years. Then it is found that the detached epicondyle is obviously out of place and is movable. Full extension may be impaired and the joint is unstable laterally. The complaint is of weakness and sometimes of ulnar neuritis set up by chronic irritation of the nerve as it passes behind the disordered lower end of the humerus. In such cases anterior transposition of the nerve will relieve the nerve symptoms, but little can be gained by interfering with the displaced epicondyle. Since by no closed method can the fracture be fixed in place these complications suggest that where practicable the method of choice is to operate and suture the epicondyle back in position. It will be found at the operation that the fragment is largely cartilaginous and that it has undergone rotation as well as downward displacement by the action of the muscles. By flexing the elbow it can be reattached in position by means of thick catgut sutures introduced by a stout curved needle, or occasionally by fixation with a beef bone peg. Flexion is maintained for three to four weeks by a plaster gutter splint, and subsequently active movements are .encouraged but no physical treatment allowed.

(c) Fractures of the Internal Epicondyle with Displacement of the Fragment into the Joint.-This type of injury is now well recognized, and is of importance not only on account of the interference with the joint mechanism but because of the frequency of ulnar nerve involvement. To permit the entry of the displaced fragment some degree of dislocation must occur.

It is sometimes associated with gross postero-lateral dislocation. The ulnar nerve may then be dragged into the joint as well and be subjected to immediate pressure. More often the nerve pressure symptoms do not occur until later on, and are then due to chronic irritation or pressure. The presence of the dislocation is usually obvious enough, but the displaced epicondyle inside the joint may not be so readily detected. An $x$-ray examination should reveal this. If an attempt be made to reduce the dislocation alone it may become apparent that there is some hindrance to complete reduction in that extension of the joint remains limited and some valgus deformity of the elbow persists. This is due to the epicondyle remaining trapped inside the joint.

$A$ lateral subluxation without complete dislocation is a more frequent occurrence. The joint is forced open on the inner side, and the avulsed epicondyle, pulled down by the muscles, enters and becomes caught up in the joint as it closes. Late cases are encountered in which the condition was not recognized at the time of the injury (see Special Plate). The signs and symptoms are very definite. Some degree of ulnar paralysis is present from the beginning. This is due to stretching of the nerve by violent abduction of the elbow. Movements, especially extension, are limited, and there is an increase in the " carrying angle," in addition to some lateral displacement of the forearm bones on the humerus. The absence of the bony prominence formed by the internal epicondyle may be detected. Opinions differ as to the best line of treatment. If the exact state of affairs is appreciated then manipulation may extricate the imprisoned epicondyle. The elbow is abducted to open the joint space on the inner side and then moved about to free the fragment. Forcible supination of the forearm with extension of the wrist and fingers may cause the epicondyle to leave the joint. The elbow is then treated in flexion. The immediate result may be satisfactory, but the question of the future must be considered. Non-union of the epicondyle will occur, and even if the initial ulnar nerve paralysis clears up a chronic neuritis may develop later. Open operation is therefore to be recommended. The ulnar nerve is first of all exposed, freed, and retracted ; the displaced epicondyle is then retrieved from the joint with as little separation of the soft tisues as possible and reattached to the lower end of the humerus. The last and most important step in the operation is to transpose the nerve to the front of the condyle, where it can lie relaxed in a muscle bed.

BIBLIOGRAPHY

Wilson, P. D.: Surg., Gynecol. and Obstet., 1933, lvi, 335

Speed, J. S.: Journ. Bone and Joint Surg., 1933, xv, 903.

Roberts, N. W.: Lancet, 1934, ii, 78, 281

Clarke, H. Osmond: Paper read before the British Orthopaedic Association, October, 1935.

G. Deligdisch (Thèse de Paris, 1936, No. 32), who records eight illustrative cases in patients aged from 2 to 37, maintains that the diagnosis of wound diphtheria cannot be made on clinical grounds alone, as the local symptoms are not absolutely typical. The membrane in particular is often produced by other organisms than the diphtheria bacillus, and is frequently absent in cases of diphtheritic infection. Cases of infection by a pure growth of the Klebs-Loeffler bacillus are very rare, and as a rule there is a mixed infection. The diphtheria bacillus has never been found in a primarily closed lesion, nor in a closed metastatic abscess arising from a focus containing this organism associated with other germs. Writers who have been content to identify the diphtheria bacillus by its morphological appearance only have found a very high percentage (50 to 60 ) of wound diphtheria, whereas those who have differentiated the bacillus by biological tests have obtained only small percentages (0 to 6). The variety of toxaemia in wound diphtheria is explained by the fact that most of the cases on record occurred in adults, who were therefore immunized in most cases. The treatment of wound diphtheria is disappointing. Most of the chemical agents suggested for local treatment have no value as their action is merely superficial; the injection of antitoxin is not always successful. 


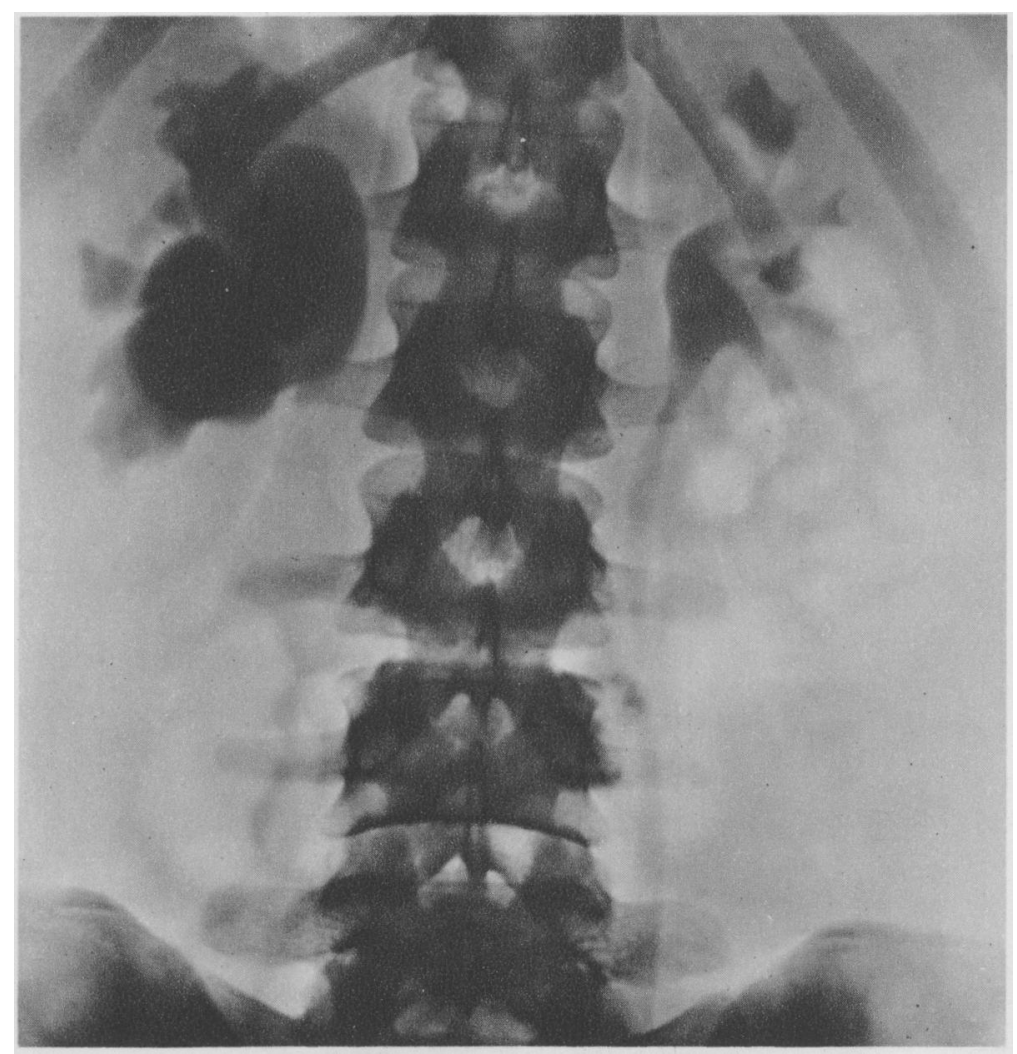

Fia. 1.-Pyelogram of Case III before operation.

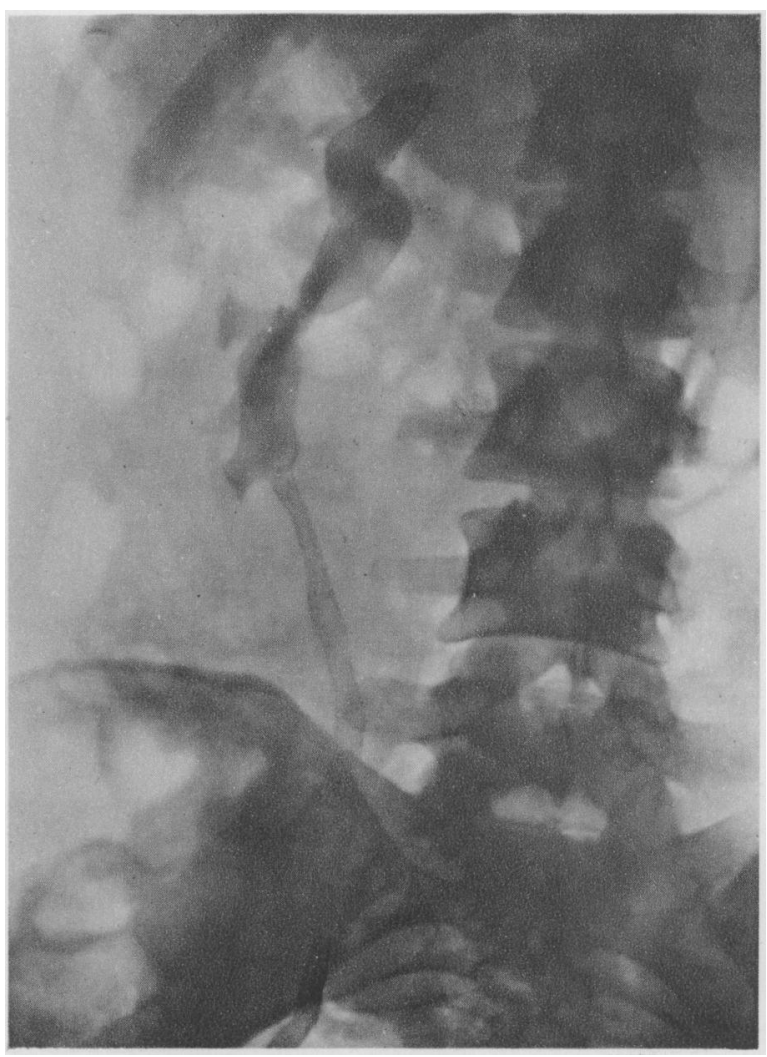

Fia. 2. - Pyelogram of Case III some weeks after operation.

\section{S. L. HIGGS : FRACTURES OF THE INTERNAL EPICONDYLE OF THE HUMERUS}

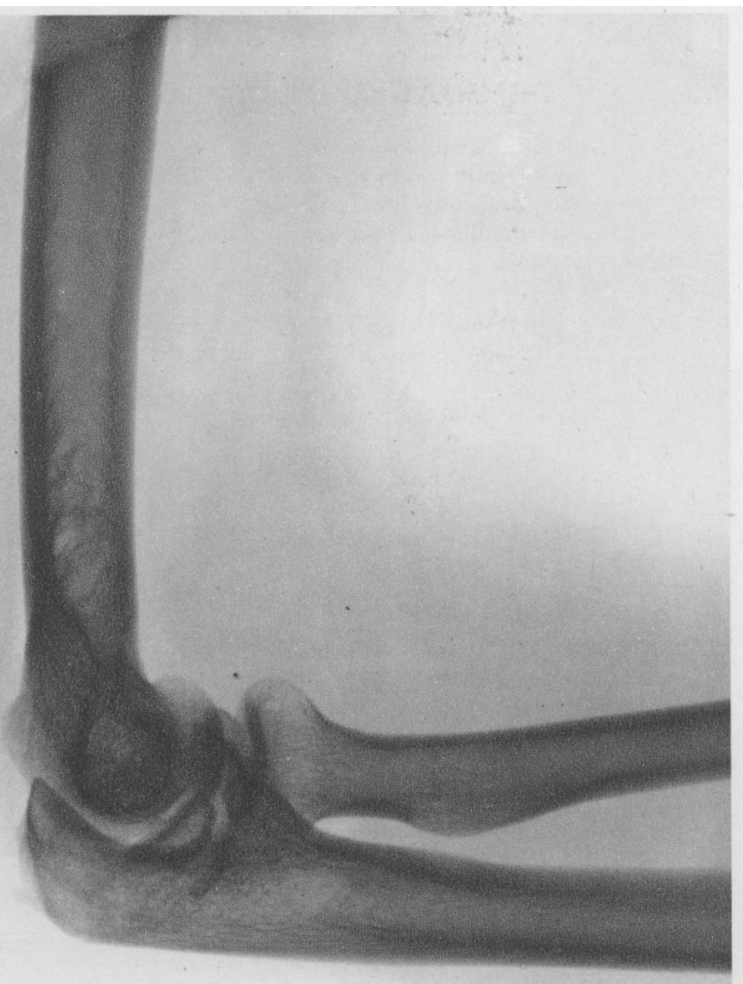

Fracture of internal epicondyle with Inclusion in elbow-Joint. The radiographs show absence of normal prominence of internal epicondyle; presence of fragment inslde the Jolnt; some cubitus valgus, and sllght outward displacement of radius and ulna. The patient had typlcal delayed ulnar neuritis. 